\title{
Kinematics Analysis and Optimization of Carbon-Free Vehicle of "8"-Shaped Trajectory Based on Spatial RSSR Mechanism
}

\author{
Chen Lei ${ }^{1,2}$, Liu Tongyi ${ }^{1, ~ *}$, Xu Tongle ${ }^{1}$ \\ ${ }^{1}$ School of Mechanical Engineering, Shandong University of Technology, Zibo, China \\ ${ }^{2}$ Institute of Instruments and Electronics, Central North University, Taiyuan, China \\ Email address: \\ 1ty-0611@163.com (Liu Tongyi) \\ ${ }^{*}$ Corresponding author
}

\section{To cite this article:}

Chen Lei, Liu Tongyi, Xu Tongle. Kinematics Analysis and Optimization of Carbon-Free Vehicle of "8"-Shaped Trajectory Based on Spatial RSSR Mechanism. Science Research. Vol. 7, No. 1, 2019, pp. 1-7. doi: 10.11648/j.sr.20190701.11

Received: January 28, 2019; Accepted: March 11, 2019; Published: March 25, 2019

\begin{abstract}
In view of the low stability of the carbon-free trolley based on the spatial RSSR mechanism, the equivalent plane mechanism method is used to analyze the spatial RSSR mechanism and the motion analysis of its driving mechanism is carried out. A fully parameterized system model is established by using the differential method to calculate the running equation of the wheel. The whole optimization method is obtained by using the calculation function of MATLAB to simulate the motion of the system, and the trajectory of the car is optimized.
\end{abstract}

Keywords: Carbon-Free Vehicle, " 8 "-Shaped Trajectory, Spatial RSSR Mechanism, Corner

\section{Introduction}

"8" zigzag track carbon-free car is the item of the national college students' comprehensive ability competition for engineering training. The car must have the automatic control and steering mechanism, and this mechanism should have the adjustable function, and only after the adjustment of the vehicle can it automatically drive the closed track. The track smoothness and running stability are determined by the steering mechanism. At present, most of the steering mechanisms designed by the " 8 " type teams are intermittent. Such as incomplete gears, grooves, etc., such as the structure of the car in operation is not high stability. To solve this problem, continuous mechanism (such as connecting rod mechanism) should be used as steering mechanism. Spatial four-bar mechanism is not only tightly structured It is flexible and reliable, so the space four-bar mechanism will be more adjustable in the non-carbon car steering design. In this study, the steering mechanism of "8" zigzag track carbon-free trolley is designed based on the spatial RSSR mechanism. The kinematics analysis of each mechanism of the carbon-free vehicle is carried out, and a fully parameterized system model is created and optimized to obtain the ideal running track.

\section{The Principle of 1-Drive and Its Optimization}

\subsection{Driving Principle Analysis}

The overall structure of the carbon-free trolley based on RSSR mechanism is shown in figure 1. From top to bottom, T is fixed on the top of the frame, and rope 1 bypasses the fixed pulley $\mathrm{T}$ to hang the weight $\mathrm{F}$. So the weight's gravity passes through the rope 1 to the drive wheel 1 at the lower end of the frame (radius is 1).), that is, the drive moment is generated on the winding axis. The torque of the drive wheel 1 is transmitted to the rear wheel through the primary gear drive (the transmission ratio of the first gear is), consisting of a fixed axis gear train 1-2-3- The crank of the spatial RSSR mechanism and the active wheel at the same time Connecting, crank Connecting rod And rocker rod A spatial crank rocker mechanism connected by two ball hinges Spatial RSSR mechanism It was through this mechanism that the car controlled the steering of the front wheel. 


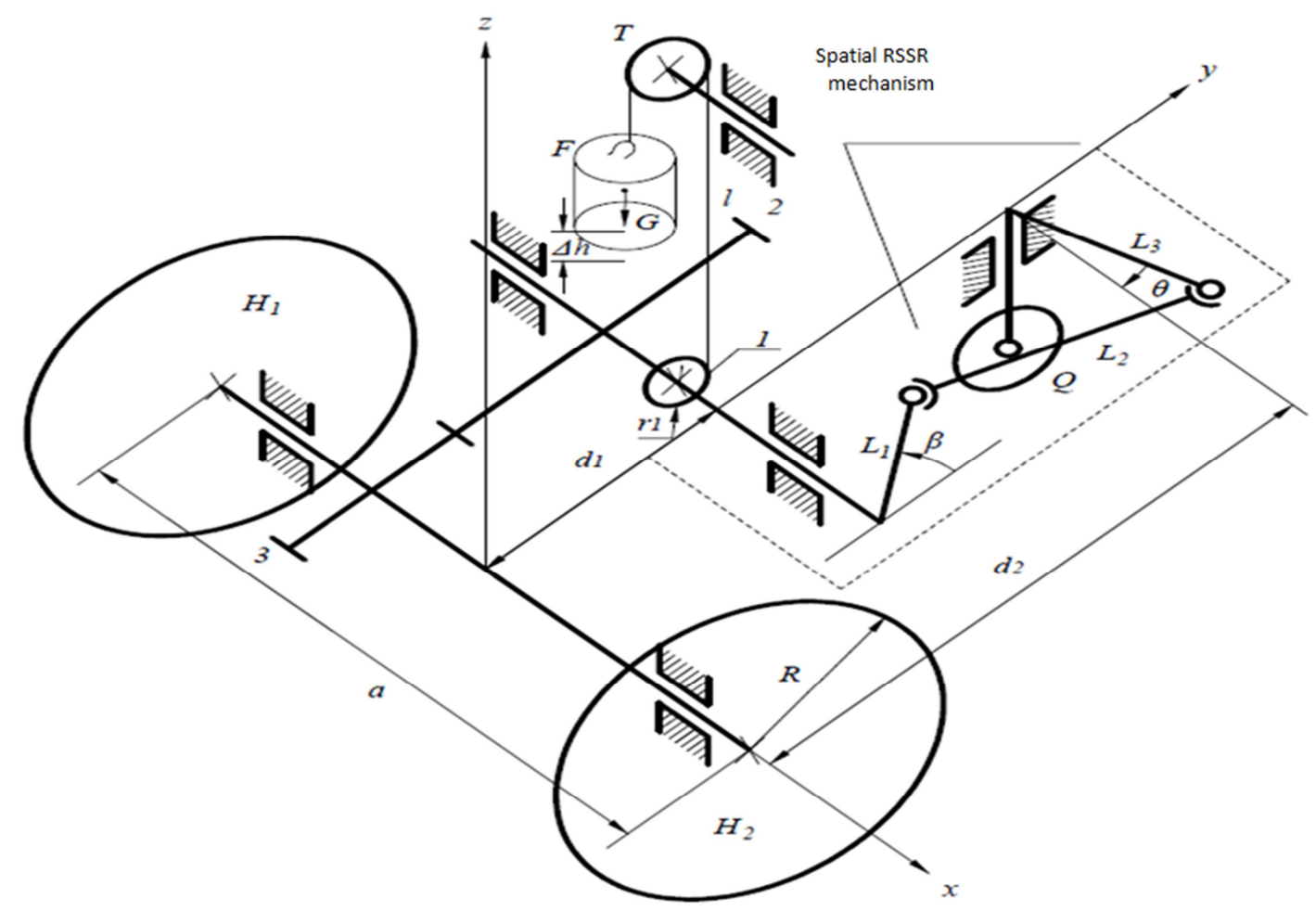

Figure 1. Overall structure of car.

\subsection{Drive Optimization}

In the past, a single wheel drive scheme was used to realize the differential motion of the left and right wheels. The single wheel drive is to connect a rear wheel directly with the drive wheel 3, and the driving torque is transferred to the rear wheel through the first gear drive. The rear wheel is always used as the driving wheel to drive the car motion. The other rear wheel adopts deep groove ball bearing as differential support from drive wheel 3 to realize differential motion. Under the single-wheel drive scheme, different driving torque is required when the vehicle is running in the left and right half periods, which will lead to the fluctuation of the running speed of the two periods, which is not conducive to the smooth operation of the car.

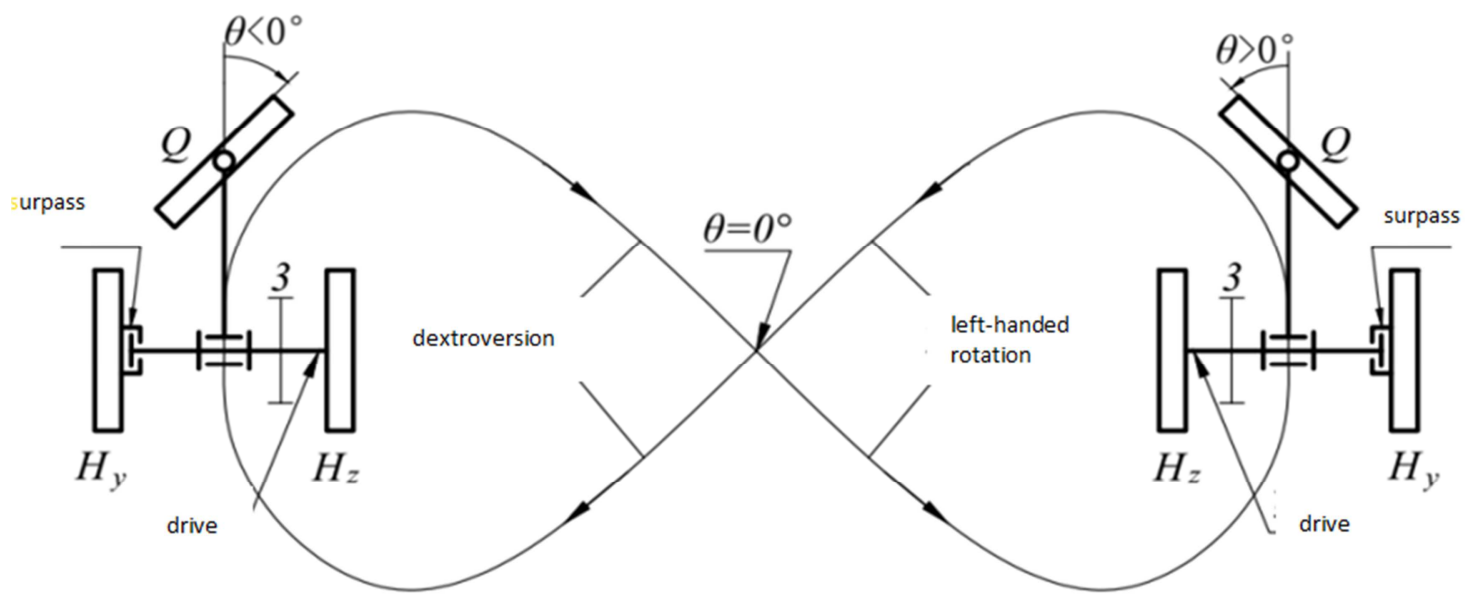

Figure 2. Two-wheel drive schematic diagram.

The two-wheel drive scheme is adopted to optimize the e system of the car. The principle of two-wheel drive is shown in figure 2. Both rear wheels use one-way needle roller bearings as overrunning clutches while supporting from the drive wheel 3. The speed of the rear wheel exceeds that of the slave wheel 3, and the speed of the slave wheel 3 does not exceed the speed of the rear wheel. When the trolley is running, the speed of the outside wheel is always greater than that of the inner wheel, so the speed of the outside wheel will exceed the speed of the slave wheel 3. The speed of the inner wheel is equal to that of the motor wheel 3, and the inner wheel is driven from the drive wheel 3 to drive the trolley. In a single cycle, the car takes the left and right wheels as the The running stage of the inner wheel is half a cycle, so the left and right 
wheels will be alternately operated as the driving wheel driving trolley when the car is running. Because the two-wheel drive further improves the stability of the car, it adopts two-wheel drive.

\section{Steering Mechanism Analysis}

In the spatial RSSR mechanism shown in figure 3, the, And The kinematic pairs are the rotational pair $\mathrm{R}$, the spherical pair
$\mathrm{S}$, the spherical pair $\mathrm{S}$ and the rotational pair $\mathrm{R}$ in turn. The carbon-free trolley uses the RSSR mechanism adopted, which is a kind of positive spatial linkage mechanism with vertical staggered follower shaft (the fork angle is equal to $90^{\circ}$ ).. The drive shaft is assembled on the winding shaft, driven by the heavy body traction rope, and the follower shaft is assembled on the front wheel steering shaft to directly control the front wheel steering.

\subsection{Kinematic Analysis Spatial RSSR Mechanism}

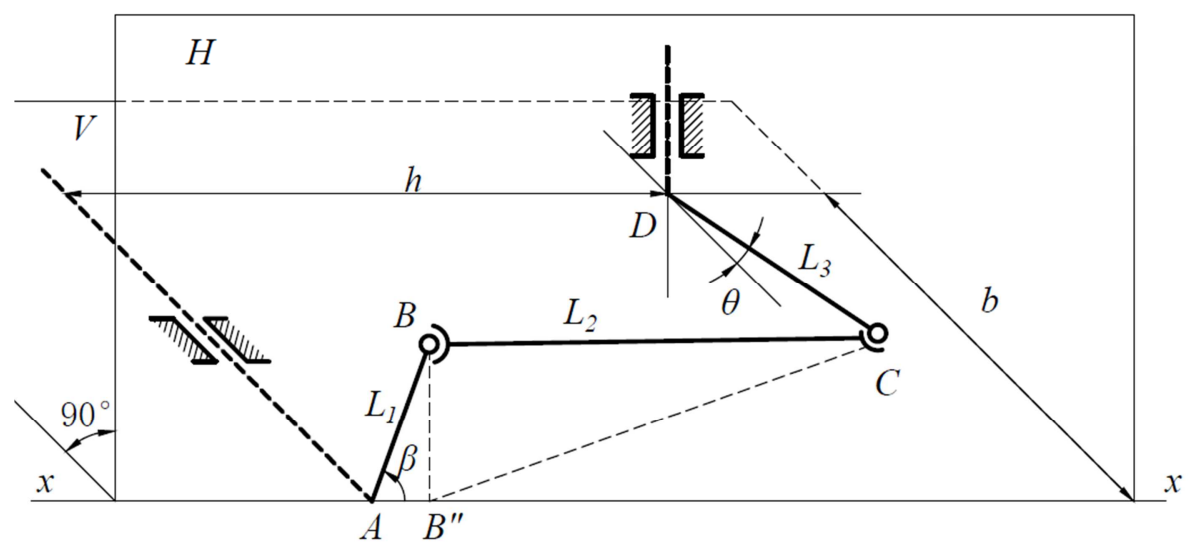

Figure 3. Spatial RSSR mechanism.

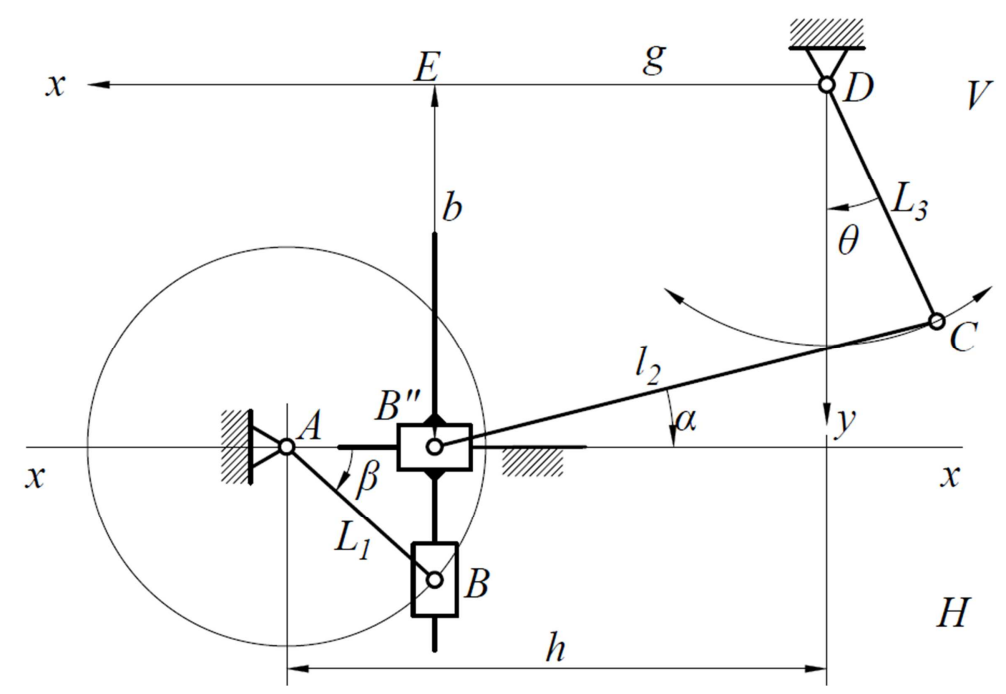

Figure 4. Equivalent plane mechanism.

The equivalent plane mechanism method is used to analyze the motion of spatial RSSR mechanism. As shown in Figure 3, the active rod $\mathrm{AB}$ and the follower $\mathrm{CD}$ constitute the rotating pair with the frame, and the master and slave shaft $A$ is in the swing plane of the follower $\mathrm{CD}$, while the connecting rod $\mathrm{BC}$ and the spherical pair are formed respectively. By doing plane $\mathrm{H}$ and $\mathrm{V}$ respectively, $\mathrm{B}$ and $\mathrm{C}$ are perpendicular to the master-slave axis $\mathrm{A}$ and the follower axis $\mathrm{B}$, respectively. The intersection line of the two planes is the projection of $\mathrm{xx} . \mathrm{b}$ point on plane $\mathrm{V}$. It must be on a straight line $\mathrm{xx}$. When the active rod $\mathrm{AB}$ revolves around axis $\mathrm{A}$, that is, point $\mathrm{B}$ is centered around $\mathrm{A}$, When the circular motion is a radius, its projection A straight line of work is performed along the $\mathrm{xx}$. So, in flat, Remote sensing slider mechanism DC in V plane Where the follower DC has a real length And the connecting rod $\mathrm{C}$ length Is variable, as shown in figure 4 , the equivalent plane mechanism of the spatial RSSR mechanism. The sinusoidal mechanism, the guide rod motion and the point of the sinusoidal mechanism are also constructed in the plane $\mathrm{H}$. Same motion. In the sinusoidal mechanism

$$
g=h-L_{1} \cos \beta
$$

In the rocker slider mechanism in $\mathrm{V}$ plane, the variable 
length of connecting rod $\mathrm{B}$ "C Obtained from the right triangle $\mathrm{BB}$ "C shown in figure 4, that is,

$$
l_{2}^{2}=L_{2}^{2}-\left(B B^{\prime \prime}\right)^{2}
$$

With $B B^{\prime \prime}=L_{1} \sin \beta$ (see figure 4) substitution gain

$$
l_{2}=\sqrt{L_{2}^{2}-\left(L_{1} \sin \beta\right)^{2}}
$$

In the $\mathrm{V}$ plane shown in figure 3 , the right hand coordinate system $\mathrm{xDy}$, is not established according to the geometry of each side of the polygon.

$$
\left\{\begin{array}{l}
l_{2} \cos \alpha=h-L_{3} \cos \theta \\
l_{2} \sin \alpha=d-L_{3} \sin \theta
\end{array}\right.
$$

Eliminate $\alpha$ and sort it out, solution

$$
h \cos \theta+d \sin \theta+J=0
$$

Solution

$$
\theta=2 \arctan \frac{d+\sqrt{d^{2}+h^{2}-J^{2}}}{h-J}
$$

In the formula $J=\frac{l_{2}^{2}-h^{2}-d^{2}-L_{3}^{3}}{2 L_{3}}$

\subsection{Transmission Characteristics of Spatial RSSR Mechanism}

The degree of freedom of the spatial RSSR mechanism is 1. The rotation angle of the driving shaft is defined as the input angle and the swing angle of the follower shaft is defined as the output angle. According to the above motion analysis, the transmission characteristics of the orthotropic spatial RSSR mechanism are obtained. $\theta=\theta(\beta)$, Finding the first derivative for $\theta(\beta)$ is obtained $\theta^{\prime}(\beta)$. Finding the second derivative is obtained $\theta^{\prime \prime}(\beta)$. They correspond to the angular displacement, the angular velocity and the angular acceleration of $L_{3}$. For example, given (in $\mathrm{mm}$ ): $L_{1}=28.7$, $L_{2}=74.1, L_{3}=38, h=74, b=40$, The transmission characteristic curve is shown in figure 5 . From this diagram, we can see, Within a period The curve $\theta(\beta)$ is about straight line $\beta=\pi$ symmetry, and the positive spatial RSSR mechanism has no quick return characteristic. Curve $\theta^{\prime}(\beta)$ and $\theta^{\prime \prime}(\beta)$ changes smoothly, without mutation, the mechanism has no impact and jump during operation, and it will not produce vibration at high speed. Therefore, the use of spatial RSSR mechanism is beneficial to the smooth operation of the trolley. The transmission characteristics of the RSSR mechanism will directly determine the track of the vehicle. The operation trajectory can be optimized by reasonable selection of the dimensions of each component.
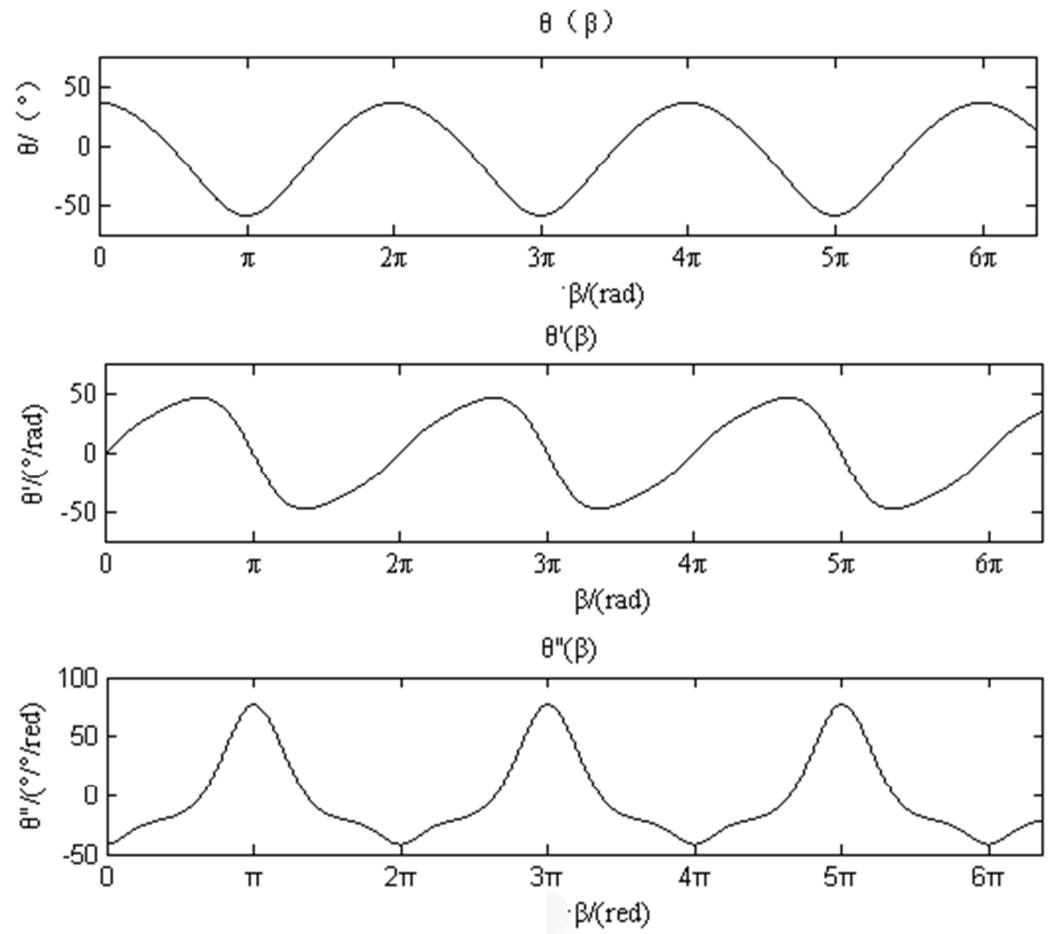

Figure 5. Transmission characteristics of spatial RSSR mechanism.

\section{Car Running Analysis}

For convenience, take the right wheel as an example to analyze its running track. For example, if the 6-graph establishes the coordinate $\mathrm{xOy}$, in a trajectory period, if the car is driven by a left wheel (inner wheel) at a position of the right half cycle $\left(\theta>0^{\circ}\right)$, The height at which the weight falls is $h$, The rotation angle of the crank is known by the formula to be The turning angle of the body relative to the ground is, The rotation angle of the crank is $\beta=h / r 1$ by the formula, The 
turning angle of the car body relative to the ground is $\phi$. The forward wheel rotation angle $\theta$ can be obtained from the transmission characteristics of the spatial RSSR mechanism.

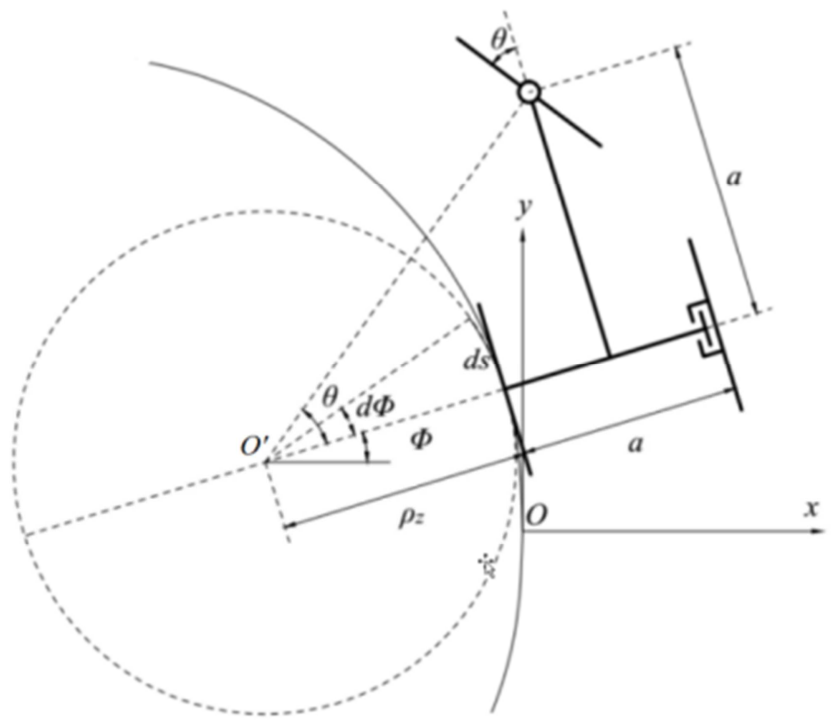

Figure 6. Kinematics analysis.

\subsection{Kinematic Analysis}

The differential element method is used to analyze, at the height $\mathrm{dh}$ at which the weight falls to a minimum Inside, the absolute displacement of the left wheel (inner wheel) is

$$
d s=\frac{d h R}{i 23 r 1}
$$

The trajectory radius [6] is obtained from the geometric relation in figure 6 is

$$
\rho_{\text {左 } z}=\frac{d 2}{\tan \theta}-a / 2
$$

The center o of the graph is the center of curvature and the center of the moment, so the absolute displacement of the right wheel (lateral wheel) is

$$
d s^{\prime}=\frac{a+\rho_{z}}{\rho_{z}} d s
$$

Replace the upper type with the $d \phi=\frac{d s}{\rho_{z}}$ and integral.

$$
\phi=\frac{R}{i 23 r 1} \int_{0}^{h} \frac{\tan \theta}{(d 2-a \tan \theta)} d h
$$

In the above formula $\theta=\theta(h)$

\subsection{Equations of Motion of Wheels}

By $\left\{\begin{array}{l}d x=-d s \cdot \sin \phi \\ d y=d s \cdot \cos \phi\end{array}\right.$ The equation of motion of the left wheel is

$$
\left\{\begin{array}{l}
x=-\frac{R}{i 23 r 1} \int_{0}^{h} \sin \phi d h \\
y=\frac{R}{i 23 r 1} \int_{0}^{h} \cos \phi d h
\end{array}\right.
$$

In the above formula $\phi=\phi(h)$

When the car is running in the left half period, the left wheel changes to the outer wheel, and its absolute displacement $\mathrm{ds}=\mathrm{ds}^{\prime}$, is the same. The equation of motion of the left wheel is obtained when the car is running in the left half period.

$$
\left\{\begin{array}{l}
x=-\frac{R(2 d 2+a \tan \theta)}{i 23 r 1(2 d 2-a \tan \theta)} \int_{0}^{h} \sin \phi d h \\
y=\frac{R(2 d 2+a \tan \theta)}{i 23 r 1(2 d 2-a \tan \theta)} \int_{0}^{h} \cos \phi d h
\end{array}\right.
$$

In the above formula $\phi=\frac{R}{i 23 r 1} \int_{0}^{h} \frac{\tan \theta(2 d 2+a \tan \theta)}{(2 d 2-a \tan \theta)^{2}} d h$

The trajectory equation of the right wheel can be obtained by geometric relation.

$$
\left\{\begin{array}{l}
x^{\prime}=x+a \cos \phi \\
y^{\prime}=y+a \sin \phi
\end{array}\right.
$$

The orbit equation of the front wheel is

$$
\left\{\begin{array}{l}
x^{\prime \prime}=x+\frac{a}{2} \cos \phi-d 2 \sin \phi^{\prime \prime} \\
y^{\prime \prime}=y+\frac{a}{2} \sin \phi+d 2 \cos \phi
\end{array}\right.
$$

\section{Motion Simulation and Optimization}

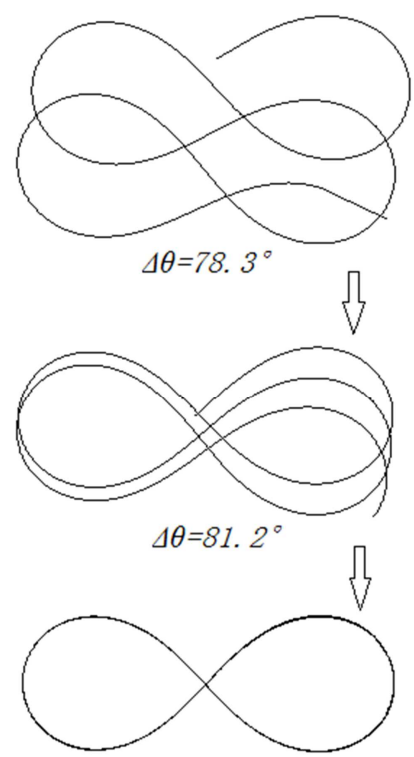

$\Delta \theta=82.5^{\circ}$

Figure 7. Simulation debugging. 


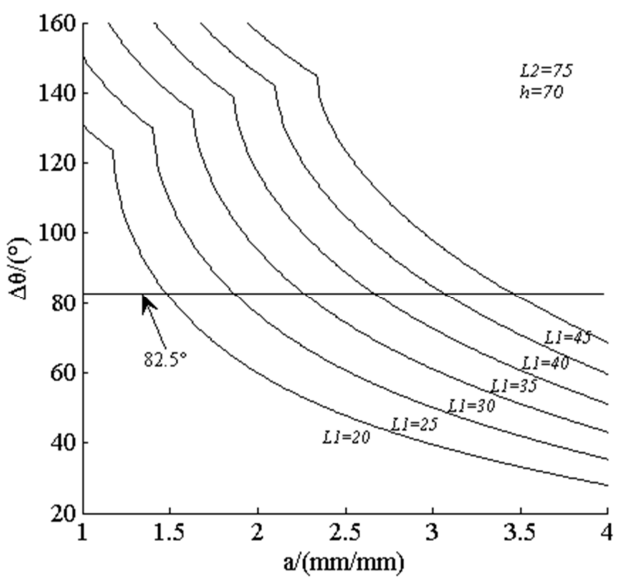

Figure 8. $\Delta \theta-a-L_{1}$ Curve.

According to the parameterized model of each mechanism of the car, the simulation track of the vehicle running can be obtained by programming the simulation system of the car in MATLAB software, assigning initial value to each parameter. ${ }^{[7]}$ However, it is necessary to adjust the initial values in order to make the simulation system simulate the "8" zigzag trajectory. Parameters in the drive system $\left(d_{1}, d_{2}, a, R, r 1, i 23\right)$ can be first determined by the design of the shape dimensions, The parameters in the steering system ( $L 1, L 2, L 3, b, h)$ multiple adjustments are needed to close the car's analog path. The adjustment process is shown in figure 7 , from this, we can see that if we want to realize the carbon-free car running according to the "8" zigzag trajectory, require swing angle of front wheel $\Delta \theta=\theta_{\max }-\theta_{\min } \geq 82.5^{\circ}$. For this, figure 8 shows the curve $\Delta \theta-a-L_{1}$, it is convenient to check the size of steering mechanism in the design of carbon-free trolley.

The specific assignment parameters and simulation tracks of each mechanism with single wheel drive are shown in tables 1 and 9, and the parameters are optimized by using double wheel drive, such as Table 2 and figure 10 .
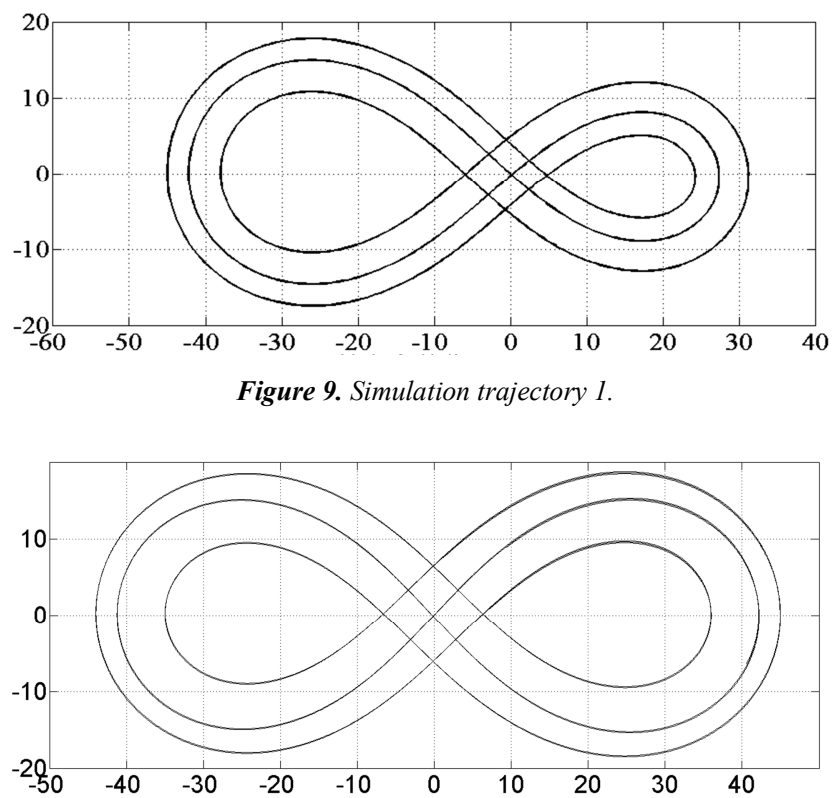

Figure 10. Simulation trajectory 2.

The trajectory is shown in figure 8 . From the track image, the curve is smooth, continuous, no jump, that is to say, the car can run smoothly. Because there is a certain error in manufacturing, the car will gradually deviate from the original path after more than one cycle. Its disadvantage is that the diameter of the track on the left and right sides is different, so it is particularly easy for the car to collide with the rod when it runs to the right. Greatly affects the number of cycles in operation.

Table 1. Assignment parameters of carbon-free vehicles.

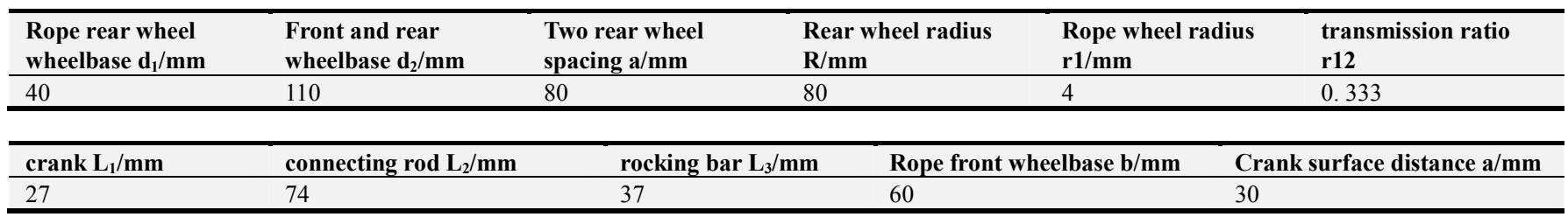

Table 2. Assignment parameters of carbon-free vehicles.

\begin{tabular}{lllll}
\hline $\begin{array}{l}\text { Rope rear wheel } \\
\text { wheelbase } \mathbf{d}_{\mathbf{1}} / \mathbf{m m}\end{array}$ & $\begin{array}{l}\text { Front and rear } \\
\text { wheelbase } \mathbf{d}_{\mathbf{2}} / \mathbf{m m}\end{array}$ & $\begin{array}{l}\text { Two rear wheel } \\
\text { spacing } \mathbf{a} / \mathbf{m m}\end{array}$ & $\begin{array}{l}\text { Rear wheel radius } \\
\mathbf{R} / \mathbf{m m}\end{array}$ & $\begin{array}{l}\text { Rope wheel radius } \\
\mathbf{r 1} / \mathbf{m m}\end{array}$ \\
\hline 43 & 113 & 90 & & $\begin{array}{l}\text { transmission ratio } \\
\mathbf{r 1 2}\end{array}$
\end{tabular}

The trajectory is shown in figure 8. From the track image, the curve is smooth, continuous, no jump, that is to say, the car can run smoothly. Because there is a certain error in manufacturing, the car will gradually deviate from the original path after more than one cycle. Its disadvantage is that the diameter of the track on the left and right sides is different, so it is particularly easy for the car to collide with the rod when it runs to the right. Greatly affects the number of cycles in operation.

\section{Conclusion}

The key points of the carbon-free car design are as follows: 1) the accurate design of the spatial RSSR mechanism. The 
spatial RSSR is the key to the automatic steering of the vehicle, including the length of crank, the length of connecting rod and the length of rocker rod. Only when these lengths are appropriate can the car walk out of a closed "8" word and return to its initial position. 2) two wheel drive differential gear. The smoothness in operation is another important performance of the trolley. Two-wheel drive solves the instability problem caused by single-wheel drive. And greatly improved the mechanical efficiency of the trolley. 3) perfect fine tuning mechanism. Due to the difficulty between the actual path and the theoretical path of the trolley. There are no errors, some minor errors can also cause the car to deviate from the track. Therefore, the assembly process requires debugging so that some of the errors can be compensated for each other. Finally, the error is reduced. So the fine tuning mechanism is the guarantee that the car can smoothly complete the "8" zigzag driving multiple times.

\section{References}

[1] Zhang Baoqing, Xiao Fuyang, Li Xiaolin, et al. Optimum Design of innovative structure for Gravity potential Energy vehicle "trajectory method" [J]. Mechanical Transmission, 2012 (3), 32 (3): 32-34.

[2] Zhang Chun, Zheng Yingbin, Ma Yongchang. Optimization of large-angle turning performance of three-wheel gravity potential vehicle [J]. Mechanical Design, 2014 (10), 31 (10): 50-55.

[3] Zhou Yong, Sun Haigang. Graphic Analysis and Design of a Type of Spatial RSSR Mechanism [J]. Journal of Beijing Institute of Technology, 2011, 04: 23-60.

[4] Miao Hongbin, Qiao Fengli. Study on Motion Analysis of Spatial RSSR Mechanism [J]. Mechanical Design and Manufacturing, 2008 (2), 2: 7-9.

[5] Lin Guangchun. A dynamic Analytical Model for a Planar adjustable 5-bar Mechanism with variable Rod length and Inertial parameters [J]. Journal of Sichuan University, 2005 (9), 37 (5): 139-143.

[6] Wang Zheng, he Flag, Hu Zeng. Design of steering Mechanism of carbon Free car based on ADAMS Software [J]. Journal of Hunan University of Technology, 2013 (9), 27 (5): 28-32.

[7] Wang Qi, Xu Shiyun, Zhao Ruitao, et al. MATLAB basic and Application examples [M]. Beijing: people's Post and Telecommunications Press, 2007.

[8] Zhang Guozhu, Wang Huigang. Design and Application of four-bar Mechanism in RSSR Space [J]. Textile Machinery, 2007 (5), 5: 36-40.

[9] Zhuang Jichao, ZongJiayi, Zhang Liqun, Liu Ziliang, Li Derong. Trajectory calculation and Analysis of 8-character carbon-free car based on Spatial RSSR Mechanism [J]. Machinery, 2017 (12), 44: 5-8.

[10] HE Yong, GU Yulian, WU Xingpei. Transformation of Spatial RSSR Mechanism to spherical 4R Mechanism [J]. Journal of Mechanical Engigneering, 2009 (10), 45: 30-34.

[11] ZHOU Xiao, SONG Meili, WANG Xiaoming, YAO Wenjin. Motion Analysis and Optimization Design of RSSR Spatial Mechanism [J]. MACHINE TOOL \& HYD R AULICS, 2015 (5), 43 (9), 7-9.

[12] Tao ZHI, wEN zHAOLIN, dING yuxing. Analytical Synthesis of Spatial RSSR Mechanism [J]. Agricultural Mechanization Research, 2001 (8), 3: 33-35.

[13] MIAO Hongbin, QIAO Fengli. Kinematics characteristics analysis research ofspatialRSSR mechanisms [J]. Mechanical Design \& Manufacture, 2008 (2), 2: 7-9.

[14] Xia xinnian. Computer aided Design of RSSR Spatial Crank-rocker Mechanism [J]. J. Wuhan Inst. Chem. Tech., 2003 (9), 25 (3): 78-81.

[15] Zhou Yong, Sun Haigang. Analysis and Design of Spatial Mechanism by Graphic Mthod [J]. Transactions of Beijing Institute of Technology, 2011 (4), 31 (4): 394-397. 